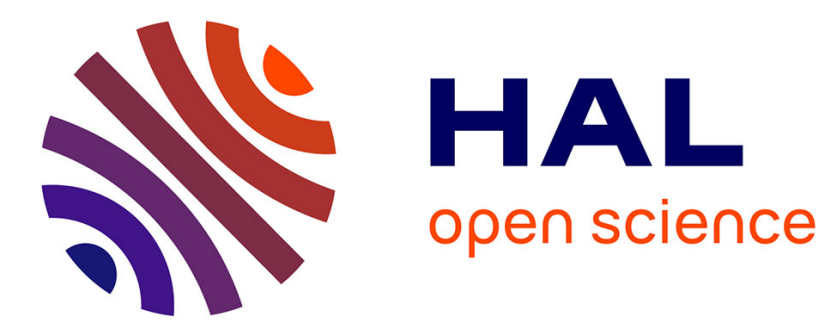

\title{
A multiscale study of the size-effect in nanoindentation of Au nanoparticles
}

Shyamal Roy, Riccardo Gatti, Benoit Devincre, Dan Mordehai

\section{To cite this version:}

Shyamal Roy, Riccardo Gatti, Benoit Devincre, Dan Mordehai. A multiscale study of the sizeeffect in nanoindentation of Au nanoparticles. Computational Materials Science, 2019, 162, pp.47-59. 10.1016/j.commatsci.2019.02.013 . hal-02382801

\section{HAL Id: hal-02382801 https://hal.science/hal-02382801}

Submitted on 9 Nov 2021

HAL is a multi-disciplinary open access archive for the deposit and dissemination of scientific research documents, whether they are published or not. The documents may come from teaching and research institutions in France or abroad, or from public or private research centers.
L'archive ouverte pluridisciplinaire HAL, est destinée au dépôt et à la diffusion de documents scientifiques de niveau recherche, publiés ou non, émanant des établissements d'enseignement et de recherche français ou étrangers, des laboratoires publics ou privés.

\section{다)(1) $(5$}

Distributed under a Creative Commons Attribution - NonCommerciall 4.0 International 


\title{
A multiscale study of the size-effect in nanoindentation of Au nanoparticles
}

\author{
S. Roy ${ }^{\mathrm{a}, \mathrm{b}, *}$, R. Gatti ${ }^{\mathrm{c}}$, B. Devincre ${ }^{\mathrm{c}}$, D. Mordehai ${ }^{\mathrm{b}, *}$ \\ ${ }^{a}$ Chair of Micromechanical Materials Modelling (MiMM), Institute of Mechanics and Fluid Dynamics, TU Bergakademie Freiberg, 09599 Freiberg, Germany \\ ${ }^{\mathrm{b}}$ Department of Mechanical Engineering, Technion - Israel Institute of Technology, Haifa, Israel \\ ${ }^{\mathrm{c}}$ LEM, UMR 104, CNRS-ONERA, 29 Av. de la Division Leclerc, Chatillon, France
}

\begin{abstract}
The mechanical response of nanoparticles is different than that of thin-films during nanoindentation tests. Moreover, it was shown experimentally that smaller nanoparticles are softer for nanoindentation. This size effect was attributed to the proximity of the free lateral surfaces to the indenter, which leads to dislocation-free surface interactions. We present here a multiscale study to show that the size effect is controlled by the interaction of the plastic zone formed beneath the indent and the lateral free surfaces. The detailed dislocation mechanisms and their interactions with the free surfaces are investigated using molecular dynamics (MD) and discrete dislocation dynamics (DDD) simulations. Au nanoparticles in the size range of 9-116 nm were indented with these two simulation techniques. The simulations show that shear dislocation loops are nucleated beneath the indent on all $\left\{\begin{array}{lll}1 & 1 & 1\end{array}\right\}$ slip planes. Dislocations interactions facilitate their escape from beneath the indent, either by forming vand u-shaped dislocations or prismatic loops that glide towards the lower part of the nanoparticles, or through glissile interactions that promote lateral dislocation motion. The effect of size on these dislocation mechanisms is discussed.
\end{abstract}

\section{Introduction}

The strengths of small-scale specimens, with dimensions on the submicrometer scale, may differ substantially from those of their bulk counterparts [1-3]. One of the experimental methods to study the effect of size and dimension on the strengths of specimens is nanoindentation [4]. While nanoindentation is a widely used technique to determine the mechanical properties of materials, it is being customarily employed in recent years to study the effect of dimensionality on the strengths of specimens on the sub-micrometer scale [5-11]. In specimens having nanoscale dimensions, where the surface-to-volume ratio is high, the free surfaces allow dislocations to escape easily from deformed specimens $[12,13]$. In pillars, for example, the size dependence of the strength was attributed to this concept of dislocation starvation. Thus, when indenting specimens with small-scale dimensions, the contribution of the free surfaces to the depletion of dislocations from the lattice yields a size effect. This can also be concluded indirectly from hardness measurements, which are performed by indenting non-flat surfaces or specimens with small-scale lateral dimensions $[6-8,14]$.

The effect of the free surfaces extends up to dimensions of a few microns, as was demonstrated for Al microwires [9]. The patterned lines, which were a few microns in diameter, were found to be softer as their lateral dimension decreased, and, in all the cases, the patterned lines were softer than those of Al thin films. Sansoz and coauthors showed through molecular dynamics (MD) simulations of Ni nanowires [15], and later, through experiments and MD simulations of Au and $\mathrm{Ni}$ nanowires [16], that the free surfaces lead to a strong softening. The effect of specimen shape also leads to a profound difference between the indentation curves of thin-films and nanoparticles of comparable heights. Chrobak et al. [17] have found through both experiments and MD simulations that dimensionality has an effect on the mode of deformation of Si specimens. The plasticity of Si nanospheres was found to be dominated by dislocations, rather than by the phase transformation occurring in bulk Si. Mordehai et al. [18] indented Au specimens and showed that the elastic responses of the nanoparticles and thin films do not differ and that plastic deformation begins at almost the same indentation depth. However, the indentation curves deviate from one another during the plastic deformation. It was proposed that the proximity of the lateral free surfaces in nanoparticles, $80-180 \mathrm{~nm}$ in height, gave rise to a reduction in the indentation force, therefore, the smaller nanoparticles were softer. They proposed that the main reason behind this size effect is the interaction of the plastic zone with the lateral free surfaces. For nanoindentation of smaller particles, the lateral free surfaces are closer to the plastic zone beneath the indent,

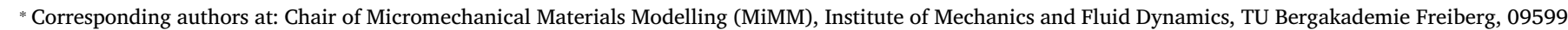
Freiberg, Germany (S. Roy); Department of Mechanical Engineering, Technion - Israel Institute of Technology, Haifa, Israel (D. Mordehai).

E-mail addresses: shyamal.roy@imfd.tu-freiberg.de (S. Roy), danmord@technion.ac.il (D. Mordehai). 
which decreases the size of the plastic zone, rendering the nanoparticles softer. An indication of the size of the plastic zone during nanoindentation can be found in [19]. Wo et al. [20] observed by using transmission electron microscopy (TEM) measurements dense plastic zones underneath the nanoindents, the radii of which were around 1.73 times larger than the radius of the contact area between the indenter tip and the specimen. The ratio can be even larger for smaller indenter tips. Zhang et al. [19] observed that the radius of a plastic zone in nanoindentation of twinning-induced plasticity steels is approximately 4 times larger than the radius of the area in contact with the indenter. This can be used to explain why the plastic zone formed during the nanoindentation of specimens with lateral surfaces is limited by the lateral size; in a larger particle, the plastic zone is less affected by the lateral free surfaces, relative to smaller particles.

To the best of our knowledge, experimentally identifying the interplay between the plastic zone and the lateral dimensions is difficult, and one may suggest the use of continuum computational models to reveal the interactions. Nonetheless, on this scale, the continuum models of plasticity break down, and it is essential to relate the plastic deformation to its basic building blocks - the dislocation microstructure and its evolution. A microstructural-based understanding of the plastic deformation involves not only investigating the complex stress field that is developed within the specimen and the various dislocation mechanisms acting beneath the indent, but also accounting for the interaction between dislocations and the free surfaces, which play a major role at the nanoscale. One of the popular computational techniques to study nanoindentation is MD simulations, which naturally captures the dislocation nucleation beneath the indent and their glides and interactions with the free surfaces. However, these simulations involve calculating the dynamics of all the atoms in the system, which practically limits the simulated specimens to a size of a few tens of nanometers. On the other hand, the mesoscale discrete dislocation dynamics (DDD) simulation technique, in which dislocation lines are the simulated entities, allows dimensions of up to a few microns [21-29]. Therefore, DDD simulations are an appealing simulation tool to study the nanoindentation of specimens that are a few hundred nanometers in dimensions.

The study of nanoindentation through DDD simulations involves a coupling with finite-element analysis (FEA) to calculate the heterogeneous elastic stress field associated with the nanoindentation load. In addition, information on the dislocation mechanisms can be extracted from atomistic simulations as part of a sequential multiscale method. Such an approach has already been employed by Fivel et al.[30-32] to investigate the nanoindentation of face-centered-cubic (FCC) bulk metals. By using molecular static simulations, they found that dislocations are nucleated beneath the indent on $\left\{\begin{array}{lll}1 & 1 & 1\end{array}\right\}$ planes and, as they glide into the bulk, they detach themselves from the indent as prismatic loops. Accordingly, dislocation prismatic loops of size that depends on the contact area between the indenter and the sample surface were introduced in the DDD simulations [32]. Other authors overcame the problem of dislocation nucleation in the simulations by considering a large density of pre-existing dislocation sources under the indenter. For instance, Po et al. [33] indented the top surface of a Cu crystal with an initial distribution of Frank-Read (FR) sources, which operated as the crystal was deformed. The hardness was calculated for both conical and spherical indenters so that comparisons with the Nix-Gao theory could be made. Gagel et al. [34] also introduced dislocations and emphasized the importance of cross-slip in the evolution of dislocations beneath the indent. They showed that cross-slip of a pinned dislocation source on a plane that is not parallel to the upper surface leads to the formation of a full-prismatic loop. A half-prismatic loop can be formed by cross-slip of the pre-existing dislocations in planes parallel to the upper surface. The formation of prismatic loops or helical loops due to cross-slip was also confirmed by other DDD simulations [35-37]. These different simulations demonstrate the benefit of using DDD simulations to study the elementary physical processes controlling nanoindentation in semi- infinite bulk materials.

Here, we use the same methodology to investigate nanoindentation in nanoparticles. Eventually, we aim to study the effects of the free surfaces bounding the plastic zone during Au nanoparticle indentation. In particular, we explore the competition between the accumulation of dislocations inside the plastic zone and their depletion on the lateral surfaces, and how this interplay is affected by the nanoparticle size. We first detail in Section 2 the MD simulations of the nanoindentation of nanoparticles of various sizes. The MD simulations allow us to discuss and identify the governing dislocation mechanisms. However, these simulations are limited by size and, in order to extend the discussion to experimental-sized particles, we extend the scale of the simulations by using DDD simulations, as reported in Section 3. We first describe the manner in which we transfer information from the MD simulation results to the DDD simulation. In the text that follows, we examine the different dislocation interactions that act within nanoparticles during nanoindentation through DDD simulations, and discuss how these mechanisms are affected by the size. Finally, in Section 4, we discuss the results obtained from the MD and DDD simulations on the role of the lateral free surfaces in the size-dependent depletion of dislocations from the plastic zone beneath the indent.

\section{Molecular dynamics simulations}

\subsection{Computational details}

MD simulations were performed by using the open source largescale atomic/molecular massively parallel simulator (LAMMPS) [38]. $\mathrm{Au}$ single-crystal nanoparticles were synthesized on a rigid substrate by using Winterbottom construction [39], similar to the work of [18]. The embedded-atom method (EAM) interatomic potential, with the parametrization proposed by Grochola et al. [40], was employed in all the simulations. The Winterbottom shape is based on the $0 \mathrm{~K}$ surface energies, which were obtained from the potential and interface energy between the Au and sapphire. The hard substrate was considered to be a lattice having the same structure and orientation as the Au nanoparticles in order to avoid mismatch at the interface, with atoms fixed at their perfect lattice positions. The interaction between the nanoparticle and the substrate atoms is based on the pair potential function of the EAM Au potential, which was modified to ensure the same work of adhesion as between $\mathrm{Au}$ and sapphire. We refer the reader to Mordehai et al. [18] for further details on the interatomic potential of the interface.

A representative nanoparticle is shown schematically in Fig. 1. The Winterbottom-shaped nanoparticle was indented by a virtual spherical indenter of radius $5.5 \mathrm{~nm}$ at the middle of the top (111) surface (see

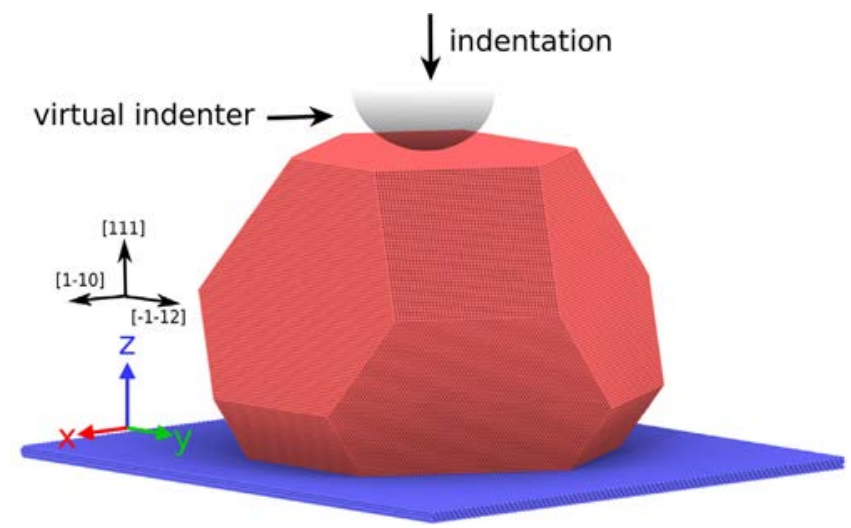

Fig. 1. Schematic description of the atomistic model. The blue-colored atoms below the nanoparticle are the fixed atoms of the substrate. Indentation was performed along the $\left[\begin{array}{lll}1 & \overline{1} & \overline{1}\end{array}\right]$ crystallographic direction by using a spherical virtual indenter of radius $5.5 \mathrm{~nm}$. 
Fig. 1). In order to isolate the effect of the lateral dimension on the plastic response, the indenter radius was kept constant for all the simulations and the size of the nanoparticle was varied from 9 to $24.2 \mathrm{~nm}$ in height. In this way, the contact between the indenter and the nanoparticles was indifferent to the particle size. The particles were first relaxed in the NVT canonical ensemble at $10 \mathrm{~K}$ for $30 \mathrm{ps}$. The time step in all the dynamic parts was set to $3 \mathrm{fs}$. Then, the particles were indented in the microcanonical ensemble at a constant displacement rate at the same temperature. In all the simulations reported here, the indenter was lowered at a constant velocity of $1 \mathrm{~m} / \mathrm{s}$ up to an indentation depth equal to $3.3 \mathrm{~nm}$. This maximum depth is both smaller than the radius of the spherical indent and avoids indents that approach the edges of the smallest nanoparticle. However, different indentation velocities of 1,10 and $100 \mathrm{~m} / \mathrm{s}$ are also discussed to examine the importance of the indentation rate on the observed dislocation mechanisms.

During the simulation, the force on the indenter and the indentation depth were recorded. Defect formation during the indentation was analyzed by using the common-neighbor analysis technique and the dislocation extraction algorithm (DXA). Both tools are parts of OVITO, which is also employed here for visualization [41].

\subsection{Indentation curves}

The force-displacement curves of all nanoparticles are shown in Fig. 2a. The elastic responses of the particles are size independent, an indication that the lateral surfaces and the interface with the substrate are far enough from the indentation point to have a minor effect on the elastic field developed around the indent. The deviation from elasticity in the force-displacement curves is related to dislocation nucleation
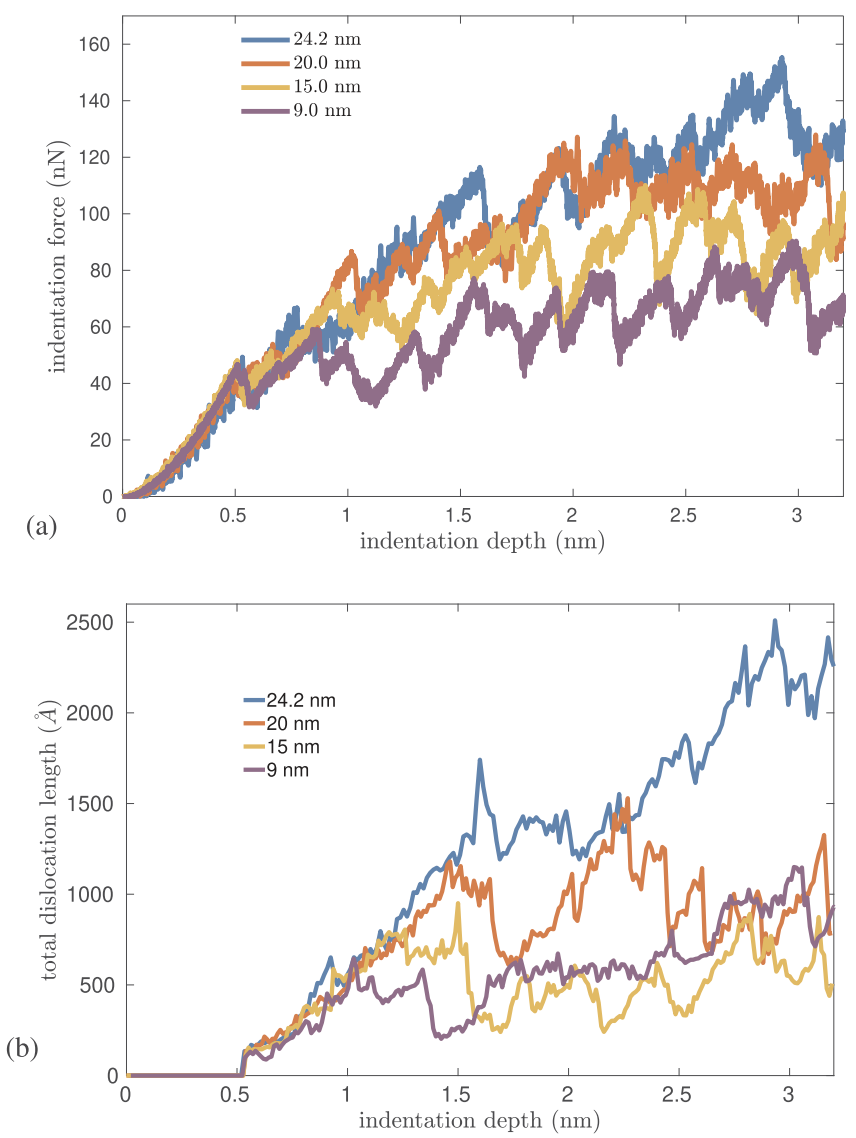

Fig. 2. (a) The MD force-displacement curves calculated for Au nanoparticles with different heights. (b) The total dislocation lengths at various indentation depths. and glide. As the indenter print increases, the applied force increases with successive load drops. Here, it must be noted that the indentation force needed to further deform the nanoparticle was found to increase on average with increasing nanoparticle size. This simulation result agrees with experimental observations [18]. For instance, the force level after indenting to a depth of $3 \mathrm{~nm}$ decreases with an increase in the nanoparticle size; for a $24.2 \mathrm{~nm}$ particle, the indentation force fluctuates around $130 \mathrm{nN}$, whereas, for 20,15 and $9 \mathrm{~nm}$ particles, these are 105,90 and $70 \mathrm{nN}$, respectively.

The elastic response is found to be size independent, while deviations between the plots can be observed once dislocations start getting nucleated. Hence, we would like to estimate the total length of the dislocations confined within the volume of the nanoparticles during the indentation. For this, we employed the DXA, counting the sum of all the dislocations and considering Shockley partial dislocations to be only half of their length. The results are shown in Fig. 2b. As the plastic deformation begins, the length of the dislocation increases for all the sizes at the same rate, and then becomes a size-dependent constant; at indentation depths of 1-2 nm, less dislocations are accommodated in the smaller nanoparticles. Following this region, larger fluctuations in the total dislocation length were observed. Nonetheless, the dislocation length in the largest nanoparticle at the final stages of the indentation was the largest. In order to better characterize the microstructural origins of the deformation, let us examine the dislocation mechanisms acting during the indentation.

\subsection{Formation of $v$-shaped dislocations}

During the initial stages of plastic deformation, dislocations are nucleated on the $\left\{\begin{array}{lll}1 & 1 & 1\end{array}\right\}$ planes in the out-of-plane directions, which are the slip planes non-parallel to the upper surfaces (Fig. 3a-b). As was observed by others [42,43], nucleation of faulted shear dislocation loops occurs slightly below the interface with the indent within the bulk. As two dislocation loops are nucleated on two different $\left\{\begin{array}{lll}1 & 1 & 1\end{array}\right\}$ planes that expand on their slip planes, one side reaches the upper surface, while the other side interacts with the second dislocation (Fig. 3b). Both the dislocations annihilate at their intersection point (collinear reaction), and the two faulted loops convert into a single fully dissociated dislocation that has a 'v-shape' and lies on the two original slip planes (Fig. 3c). We conclude from this reaction that both the dislocations have the same Burgers vector (but with opposite line senses at their intersection point), probably since it is a geometrical necessity to accommodate the penetrating indent. This v-shaped dislocation now glides away from the indent and, thus, expands while having its two open ends pinned at the top surface of the indent.

As the deformation proceeds, more dislocations are nucleated and similar v-shaped dislocations form and glide away from the indent in all three out-of-plane directions (Fig. 3d). Additionally, dislocations are nucleated on planes parallel to the upper surface. In all the simulations, these additional faulted loops, which are close and parallel to the upper surface, always appeared after the nucleation of the out-of-plane dislocation loops. These dislocations, which we denote as in-plane dislocations, glide parallel to the top facet, as shown in Fig. 3d. Similar inplane dislocation loops were identified in $\mathrm{Cu}$ [44].

In between the load drops, the indentation stress increases, pushing the front of the v-shaped dislocation in the direction of the Burgers vector and towards the lower parts of the nanoparticles. During this time, the two pinned ends may be released one by one or across a very short time interval, as demonstrated in Fig. 4. However, if the particle is small enough, these $v$-shaped dislocations may reach the lateral surfaces before getting unpinned (Fig. 5a-b). The smaller the lateral dimension of the nanoparticles, the higher is the probability of the dislocation reaching the lateral free surfaces before getting unpinned. The process of formation of $\mathrm{v}$-shaped dislocations and their annihilation at the free surfaces is demonstrated for smaller particles in Supplementary Movies 1 and 2. 


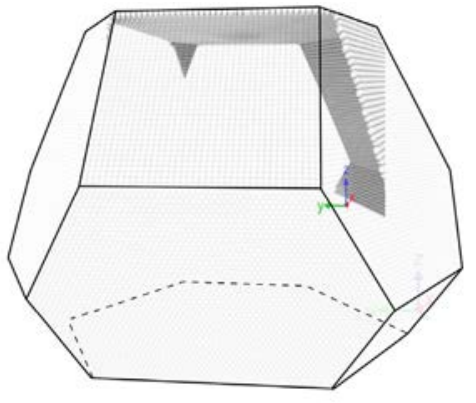

(a)

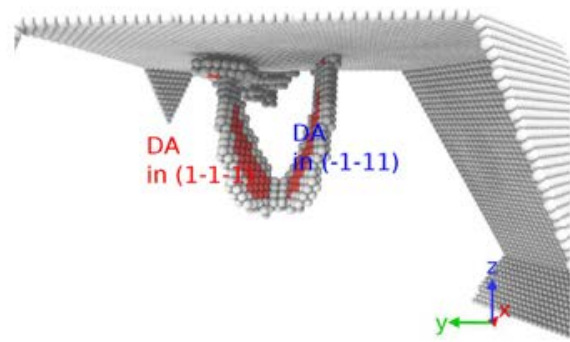

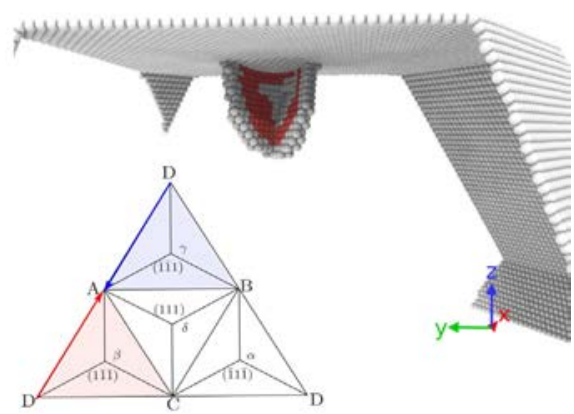

(b)

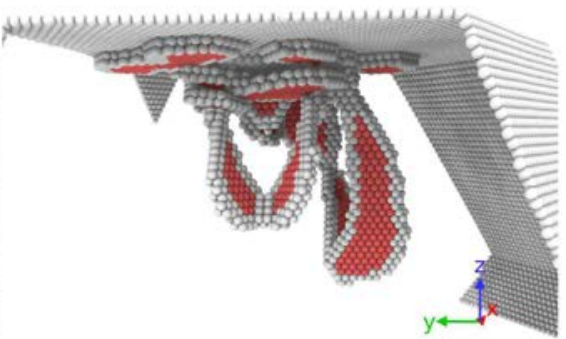

Fig. 3. A few initial dislocation nucleation events in a $20 \mathrm{~nm}$ high particle. (a) The region where indenter-particle contact and possible dislocation nucleation occur inside the particle is highlighted. (b) Two out-of-plane dislocations are nucleated beneath the indent on two different slip planes. They have the same Burgers vectors, but these are in opposite directions. (c) The two out-of-plane dislocations engage in a collinear reaction and annihilate at the intersection point, forming a v-shaped dislocation. (d) Two additional out-of-plane dislocations are nucleated that form another v-shaped dislocation after a collinear reaction. Some faulted loops of depth one atomic layer and some dislocations in the in-plane direction are later nucleated parallel to the top surface. (c)

(d)
The annihilation at the lateral surfaces leaves behind two dislocation segments between the pinning points on the upper surface and those on the lateral surface (Fig. 5b). Once the dislocation reaches the surface, its line tension acts to straighten the dislocation in order to decrease its energy. Then, the remaining dislocation segments rapidly glide and annihilate at the surface (Fig. 5a-c). Interestingly, the annihilation of these segments at the surfaces is accelerated only when the $\mathrm{v}$-shaped dislocations touch the free surfaces. This leads us to the conclusion that the main contribution of the free surfaces to the dislocation motion is the contact interaction between the dislocations and the free surfaces, rather than the image forces acting on the dislocations inside the nanoparticle before they touch a free surface. As we shall further discuss, we argue that the contact interaction between dislocations and free surfaces is the main contribution of the surfaces, which is also considered in the DDD simulations. In addition, it is worth pointing out that the surface steps created by the out-of-plane dislocations while annihilating at the surfaces appear only in the lower part of the particle.

Other simulations of thin films reported in the literature $[11,31,45,46]$ pertain to the nucleation of prismatic loops in the out-ofplane directions, rather than the nucleation of v-shaped dislocations. One possible reason for the loop formation observed in MD simulations is the strain rate. When performing the indentation at various velocities,
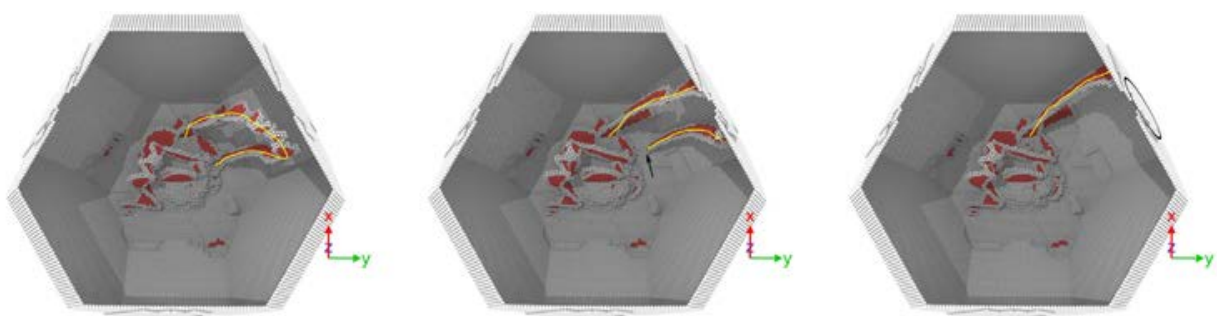

(a)

(b)

(c) we observed the formation of prismatic loops only at higher indentation rates and in the largest particles. When the particle size is large, the vshaped dislocations have enough space to grow into the bulk. Ultimately, when the indentation rate is relatively high, it allows the two upper branches of the dislocation, which are pinned at the top surface, to cross-slip into two different slip planes and glide towards one another. When these cross-slipped segments meet, they annihilate at the intersection point and a collinear reaction occurs, resulting in the formation of a prismatic loop. These prismatic loops glide in the direction of the Burgers vector and annihilate in the bottom part of the lateral surfaces, leading to a similar pattern of atomic steps on the surface as is the case with the v-shaped dislocations. For brevity, we do not show here these simulation results. Nonetheless, since the formation of prismatic loops is favored at higher indentation rates, we chose the lowest indentation rate out of the three examined here.

\subsection{Formation of dislocations in the in-plane direction}

As mentioned in Section 2.3, faulted dislocation loops are nucleated on planes parallel to the upper facet. Since the resolved shear stress arising from the external load alone is small, they are not nucleated at the early stages of the indentation, but after the nucleation of a few outof-plane dislocations. These faulted loops are nucleated only a few

Fig. 4. Annihilation of v-shaped dislocations. (a) A nanoparticle is viewed from the bottom during indentation. A v-shaped dislocation is highlighted by a line. We emphasize that some cross-slip occurred along the dislocation line. (b) One end of the v-shaped dislocation is unpinned (marked with an arrow) and glides towards the lateral surface, while the other end is still pinned. (c) A surface step (encircled in the figure) is formed on the lower part of the nanoparticle and a dislocation segment is left behind on a single slip plane within the nanoparticle as a reminiscence to the $\mathrm{v}$-shaped dislocation. 


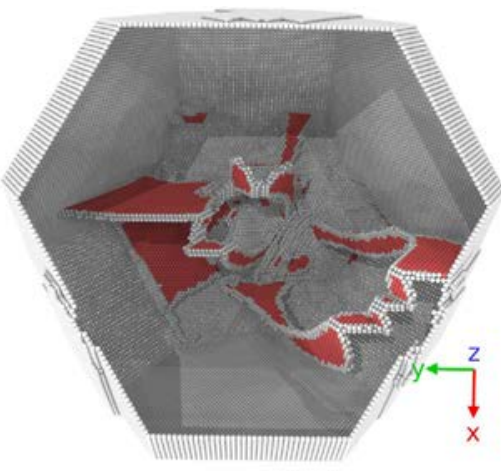

(a)

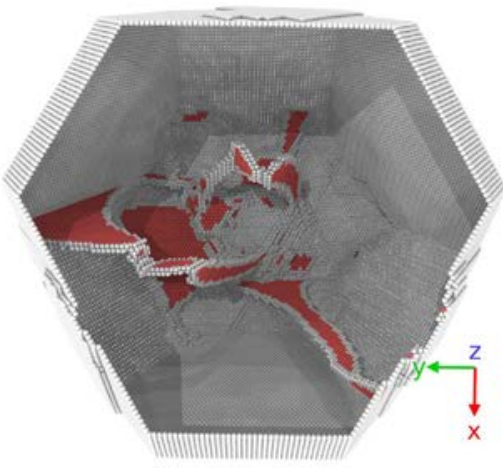

(c)

(d)

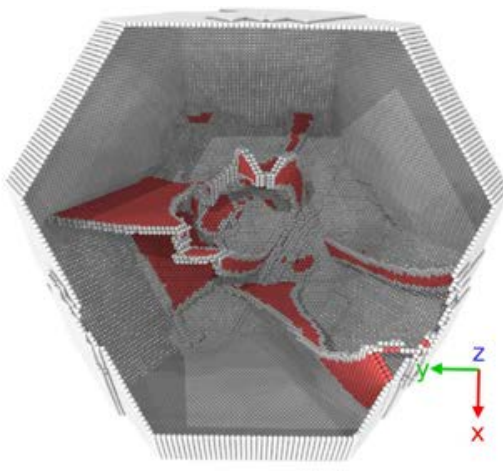

Fig. 5. Dislocation starvation in a $20 \mathrm{~nm}$ high particle, as viewed from the bottom of the nanoparticle. (a) Several v-shaped dislocations are visible in the particle, out of which one of them reached the free surface in the lower part of the particle. (b) A large $\mathrm{v}$-shaped dislocation is escaping at a free surface, leaving behind two dislocation segments in the bulk. (c) One of the segments left behind is annihilated at the free surface. (d) The v-shaped dislocation fully escapes the nanoparticle, leaving behind an atomic island on the lateral facet. A similar process occurred involving the other v-shaped dislocations visible in (a). (b)

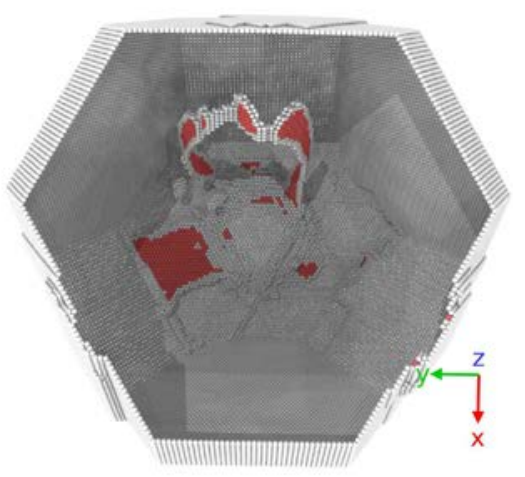

monolayers beneath the upper surface and barely glide. As a result, these loops accumulate beneath the indent and do not contribute to the deformation on the lateral surfaces as much as the v-shaped dislocations do (Fig. 3a).

Half-prismatic loops are also observed around the indent during indentation. These dislocations glide parallel to the top surface and exit at the lateral surfaces in the upper part of the particle. These halfprismatic loops allow lateral transfer of material to the upper part of the lateral surfaces. Although it is hard to track precisely the genesis of these loops, it seems that they are nucleated at the interface between the indenter and the nanoparticle. Thus, these half-loops exhibit a strong correlation with the indentation depth.

However, at the later stages of the deformation, we identified an additional mechanism that allows the material to be driven laterally via dislocations in the in-plane direction. This mechanism is a result of an interaction between two dislocations that are stored inside the plastic zone beneath the indent and gliding in different slip directions. For instance, if we adopt the coordinate system shown in Fig. 1, by using the Thompson notation, we demonstrate in Fig. 6 how the interaction between two out-of-plane dislocations results in a dislocation with a Burgers vector parallel to the upper surface:

$\mathbf{B D}+\mathbf{D A}=\mathbf{B A}$.

This glissile interaction is a reminiscence of a glissile junction. There is, however, a crucial difference: the reactant dislocation is located on neither of the slip planes of the interacting dislocations. For instance, the dislocation $\mathbf{B D}$ is either dissociated on the (1 111$)$ plane $(\mathbf{B} \alpha+\alpha \mathbf{D})$ or the $\left(\begin{array}{lll}1 & 1 & \overline{1}\end{array}\right)$ plane $(\mathbf{B} \gamma+\gamma \mathbf{D})$. DA is either dissociated on the $\left(\begin{array}{lll}1 & 1 & \overline{1}\end{array}\right)$ plane $(\mathbf{D} \gamma+\gamma \mathbf{A})$ or on the (lill 11$)$ plane $(\mathbf{D} \beta+\beta \mathbf{A})$. However, the reactant $\mathbf{B A}$ is dissociated on the $\left(\begin{array}{lll}1 & 1 & 1\end{array}\right)$ plane $(\mathbf{B} \delta+\delta \mathbf{A})$. In the case of glissile junction formation, BA should be on ( $\overline{1} \overline{1} 1)$ plane. The reason that the interaction results in a dislocation on a third slip plane (1 111$)$ is not fully clear, but we recall that the two dislocation lines entering the junction also reside on two planes, which indicates that constrictions along the dislocation lines allow cross-slip. Thus, we believe that

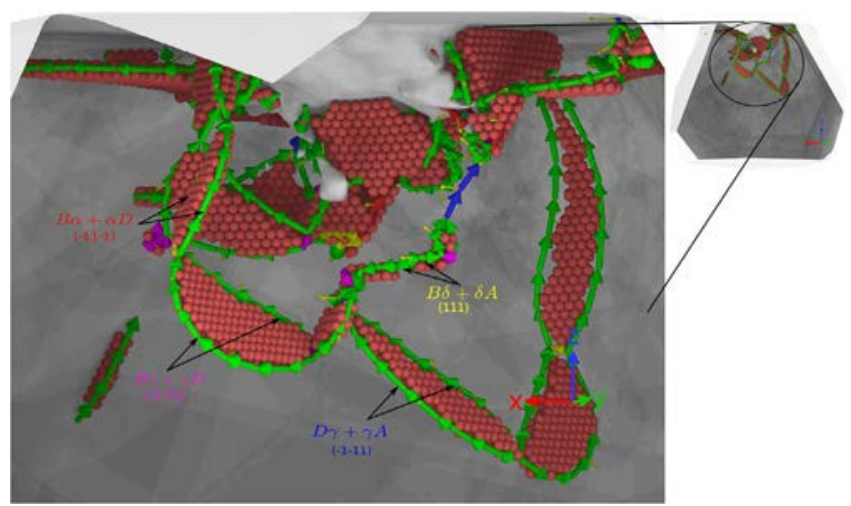

Fig. 6. Glissile interaction; the two out-of-plane dislocations BD and DA on two

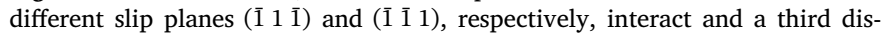
location BA on plane (1 111 ) emerges from the junction, the Burgers vector of which is parallel to the top surface. The notations are as per the Thompson tetrahedral shown in Fig. 3.

constricted screw segments in the junction drive the reactant dislocation into the third slip plane through cross-slip under the indentation stress. However, we postpone further exploration of the origin of this mechanism to the future.

Once the reactant dislocation is formed in the in-plane direction, its length increases until one of its ends reaches the upper surface. Then, this in-plane dislocation segment glides laterally towards the lateral surface, annihilating there. Eventually, the in-plane dislocation escapes the crystal from the upper part of the nanoparticle. It is worth noting that its size is larger than the size of the half-prismatic loops that are nucleated at the early stages of the indentation.

\subsection{Nanoparticle surface deformation}

The different dislocation mechanisms reported above plastically 

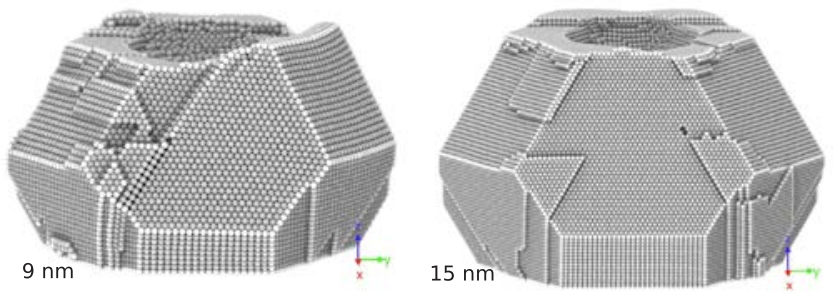

The contribution of dislocations paralle to top surfaces or half prismatic loops

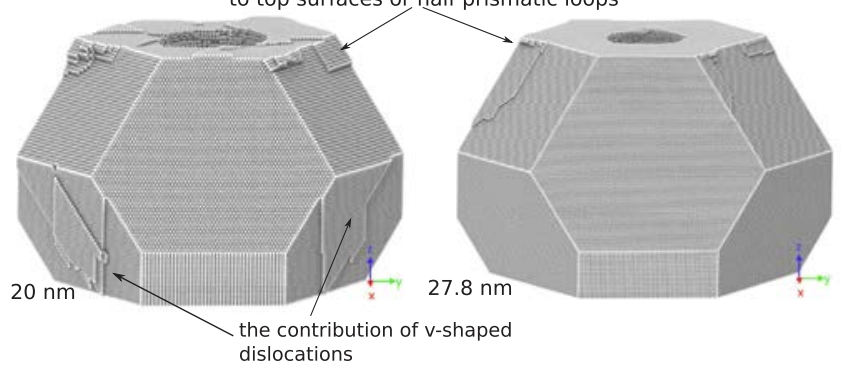

Fig. 7. Surface deformations of particles of size $9 \mathrm{~nm}$ (top-left), $15 \mathrm{~nm}$ (topright), $20 \mathrm{~nm}$ (bottom-left) and $27.8 \mathrm{~nm}$ (bottom-right) at $2.55 \mathrm{~nm}$ indentation depth. For clarity, all the particles are scaled to the same size, and the different scales can be identified from the indent imprint size. The surface deformations are characteristic of the different mechanisms observed during the indentation (v-shaped dislocations, prismatic loops, in-plane dislocations). See text for more details.

drive material away from the region beneath the indenter. Some of the dislocations escape through the lateral surfaces, leaving behind atomic steps on the nanoparticle surface. A close examination of the lateral surface (Fig. 7) reveals how the different dislocation mechanisms reported above result in different "signatures" on the surfaces.

As can be seen from Fig. 7, the surfaces of the nanoparticles are deformed, due to dislocations escaping the volume. A comparison between nanoparticles is performed at the same indentation depth. The surface of the $9 \mathrm{~nm}$ high particle is severely deformed, and one can also identify substantial shearing of the nanoparticle along an out-of-plane direction. This shearing is a result of shear dislocation loops that were nucleated but did not have enough volume to participate in a collinear reaction, resulting in a dislocation residing between the lateral surfaces and gliding downwards (see Supplementary Movie 1). Since this type of deformation was observed in the smallest particle only, we do not further elaborate on this shearing. In the larger nanoparticles, we can distinguish between the typical types of atomic terraces on the lateral surfaces in the lower and upper parts of the particles.

As mentioned earlier, the v-shaped dislocations and the prismatic loops that are formed in the out-of-plane directions are mainly responsible for the deformation observed in the lower part of the particle. These dislocations glide easily and are formed in the lower part of the plastic zone underneath the indenter. The deformation in the upper part arises from the glide of dislocations in the in-plane direction. One can see from Fig. 7 that there are numerous surface steps in the upper part, which are an indication of the number of in-plane dislocations reaching the lateral surfaces. Given the fact that most of the attention in the literature has been paid to the out-of-plane dislocations, and, in particular, to the prismatic loops in the out-of-plane directions, the contribution of the in-plane dislocations is overlooked. One can often see traces formed by the in-plane dislocations that can be tracked to as low as half the particle height. This is an indication that a large portion of the glissile interactions that take place involve the out-of-plane dislocations, as described in Section 2.4.

Another indication for the extent to which dislocations escape from the nanoparticle is the amount of terraces formed on the nanoparticle surface. Although the surfaces of all the nanoparticles are compared at the same indentation depth (i.e., the same volume has been "pushed" by the indenter), the surface of the largest nanoparticle is only slightly deformed with respect to those of the smaller ones. This result is qualitatively compared with the result shown in Fig. 2b, which reveals that the total dislocation length accumulated in the larger nanoparticle is the largest.

The MD simulations provide great information on the dislocation dynamics and reactions taking place in the plastic zone underneath the indenter. It is clear from the MD simulation results that the lateral free surfaces play a major role in depleting the nucleated dislocations, which in turn affect the force-displacement curves. However, the size of nanoparticles in the MD simulations is one order of magnitude smaller than that of the experimental ones. While reaching the experimental dimensions is impractical in MD simulations, DDD simulations allow nanoparticle dimensions of the order of those observed in the experiments. In the next section, we examine how dislocation dynamics and interactions operate in larger nanoparticles by using the open source DDD code microMegas [47]. Since the DDD simulation introduces some approximations, such as considering dislocations as being constricted rather than dissociated, rules for dislocation nucleation and their interaction with free surfaces should be introduced. Accordingly, we first adjust several parameters in the DDD simulations based on the values and mechanisms obtained from the MD simulations.

\section{Discrete dislocation dynamic simulations}

\subsection{Computational methods}

We performed all the DDD simulations in two parts. The stress distribution in the indented nanoparticle was initially calculated by a finite element (FE) simulation, which was then incorporated as an applied stress field in the DDD simulations. With the ABAQUS FE solver, a Winterbottom-shaped nanoparticle was first loaded by a rigid spherical indenter at a constant velocity in a displacement controlled manner (shown in Fig. 8). Hexahedral nonlinear quadratic elements were used to mesh the nanoparticle and anisotropic elasticity was considered. The mesh at the bottom of the nanoparticle was fixed to mimic a hard substrate, and the particle orientation was such that the (1 111 ) direction was normal to the top surface. As dislocations are known to nucleate near the indent, the FE mesh was made finer near to the contact area between the nanoparticle and the indenter to accurately capture the stress field gradient in this region. Then, the stress components at all the node points of the FE mesh could be saved only at the maximum indenter depth. Such data was later tabulated and interpolated with linear shape functions to define the elastic stress field everywhere in the nanoparticles and at any indentation depth.

DDD simulations have been performed with the open source code microMegas $[48,47]$. A 3D convex domain defining the contour of the simulated particle was created inside the simulation box by defining fourteen planes with crystallographic orientations, which is consistent with Winterbottom nanoparticle shape. Dislocation dynamics were allowed only inside this domain. One of the 14 planes that is at the bottom of the domain was rendered impenetrable to dislocations in
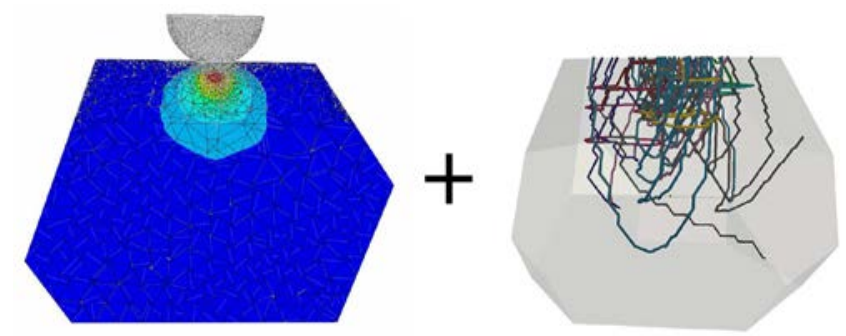

Fig. 8. Schematic drawing of the coupling between the FE and the DDD simulations used to model the loading imposed by a rigid spherical indenter on the top surface of a nanoparticle. 
order to mimic the particle-substrate interface. The other 13 planes are free surfaces; the dislocation line passing through such boundary planes are automatically annihilated. The mechanical properties associated with the image forces induced by the free surfaces were accounted for in the simulations by following the procedure first proposed by Schwarz and coauthors for modelling the dislocation dynamics in thin films and micropillars $[49,50]$. The heterogeneous loading associated with the nanoparticle indentation was calculated from the results of the FE simulation mentioned above by following the interpolation procedure defined in [51]. During the DD simulations, this loading stress was superposed with the internal stress associated with the existence of dislocations inside the nanoparticle. For simplicity, the particle contour was fixed during the DDD simulations.

Since the nanoparticles considered in the experiments and the MD simulations were initially pristine, a dislocation nucleation criterion was used in the DDD simulation to introduce dislocation loops at preferable nucleation sites and slip systems [51]. Liu et al. [52] showed that the dislocation nucleation can be implemented at preferential positions in the mesostructures, depending on the stress distribution in that region, which by itself decides whether a dislocation should be nucleated or not, and whether it will glide based on the Peach-Koehler forces acting on the dislocation line. The dislocation nucleation criterion that we used was implemented through the following steps:

1. A nucleation site and a slip system were randomly selected inside a region identified as a potential nucleation region, e.g., the region with the highest stress level.

2. A virtual octagon loop of a given inscribed size was introduced at the chosen site and slip system.

3. If the average Peach-Koehler forces on the loop acted towards expanding the loop and if at least 6 segments moved in the direction of expansion, the octagon loop was introduced at the tested site, and the subsequent steps followed the common rules of dislocation dynamics in the simulation. The DDD simulation then proceeded in time and no additional dislocations were nucleated within a certain waiting time.

4. If nucleation was unsuccessful, step numbers 1 to 3 were repeated. If, after a given number of nucleation attempts, a dislocation was not nucleated, the DDD simulation progressed through one time step.

These nucleation rules introduce a few simulation parameters that determine the nucleation rates and sites, which were adjusted in the following manner:

\subsubsection{Initial loop radius}

Several simulations have been performed under a constant homogeneous resolved shear stress, where the initial dislocation loop size was increased until an effective attempt to introduce an expanding dislocation loop was accomplished. In Fig. 9, the variation in the critical dislocation loop radius with increasing homogeneous resolved shear stress is reported.

Through such preliminary simulations, we make sure that the local nucleation rules employed in the simulation satisfy the usual equation for the nucleation of circular dislocation loops [53]:

$\tau_{c}=\frac{G b(1-\nu / 2)}{4 \pi r_{c}(1-\nu)}\left[\ln \left(2 r_{c} / r_{0}\right)+1\right]$,

where $G, v$ and $r_{0}$ are the shear modulus, Poisson's ratio, and dislocation core radius, respectively. For the elastic constants of Au considered in the DDD simulation, $G=30 \mathrm{GPa}$ and $\nu=0.44$, the best fit to the DDD simulations is achieved if the dislocation core radius is $r_{0}=1.15 b$ (Burgers vector of $\mathrm{Au}$ is $b=0.288 \mathrm{~nm}$ ). This value is in very good agreement with the theory considering the coarse line discretization that we used in these simulations. From the experimental results [54], the embryonic loop is expected to be nucleated at the atomic level when the resolved shear stress is $1.3 \mathrm{GPa}$, which corresponds to a critical loop

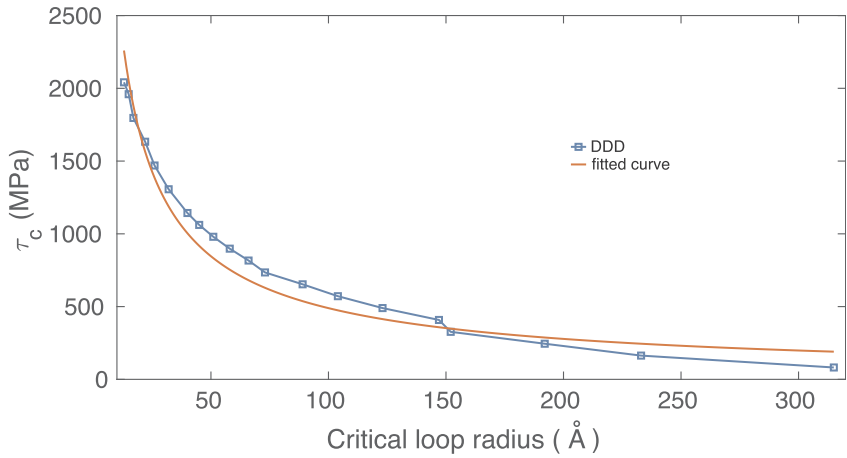

Fig. 9. Comparison between the simulated critical radius for octagon dislocation loops as a function of an applied homogeneous resolved shear stress and the result expected from classical nucleation theory (Eq. (2)).

radius of $3 \mathrm{~nm}$ (Fig. 9). Similar values can be obtained from the present MD simulation results. Based on Fig. 2, the first partial dislocation nucleation occurs at an indention depth of $0.5 \mathrm{~nm}$ and an indentation force of $50 \mathrm{nN}$, which corresponds to a projected contact area and a mean pressure of $16.5 \mathrm{~nm}^{2}$ and $3.0 \mathrm{GPa}$, respectively. According to Hertz theory, the maximum shear stress is expected to be found beneath the indent, and is equal to $46.5 \%$ of the mean pressure, i.e., $1.41 \mathrm{GPa}$. However, we recall that in the MD simulations the leading partial dislocation was nucleated, while the stress in the DDD simulations corresponds to the nucleation of a perfect dislocation (both leading and trailing partial dislocations). The ratio between the nucleation stresses of the leading and trailing partials was not measured, to the best of our knowledge. Nonetheless, the ratio between the Schmid factors, when compressed along the $\left\langle\begin{array}{llll}1 & 1 & 1\end{array}\right\rangle$ direction, is approximately equal to $0.3 / 0.25=1.2$, which is obtained from the analysis presented in Ref. [55]. Thus, considering the same value for the nucleation stresses of both the leading and trailing partials, we expect a full dislocation to be nucleated at the shear stress of $1.7 \mathrm{GPa}$. This stress corresponds to a critical loop radius of $1.5 \mathrm{~nm}$ (Fig. 9) in the DDD simulations. Therefore, we choose this value as the nucleation threshold. Additionally, it is kept constant in all the simulations, as the indenter radius and the critical nucleation stress level were observed to be the same in the MD simulations as well as in the experiments.

\subsubsection{Waiting time}

A waiting time between two nucleation events was introduced in order to avoid very fast nucleation of consecutive loops at the same site, which results in extensive dislocation nucleation on the same slip plane. This value was chosen based on observation of the MD simulations. The average time between two nucleation events over the first 5-6 nucleation events in the MD simulation was found to be $\approx 1$ ns for an indenter velocity of $1 \mathrm{~m} / \mathrm{s}$. Since the indentation rates in DDD are smaller, we expect the value obtained from the MD simulations to be an upper limit. Therefore, the value of $0.3 \mathrm{~ns}$ was considered in all the DDD simulations. It is worth noting that the nucleation rate in the DDD simulations further presented was comparable with similar observations made elsewhere [31,32,56].

\subsubsection{Nucleation location}

It is obvious that dislocation nucleation is substantially more likely to occur in a region where the stress levels are high. Therefore, nucleation was allowed only in a smaller volume within the nanoparticles to optimize the random sampling of the nucleation sites. The shape and dimensions of the nucleation box were kept constant for all particle sizes, as the shape and size of the indenter were the same. The height of the cubic nucleation box was $15 \mathrm{~nm}$, which was $65 \%, 22 \%$ and $13 \%$ of the smallest, medium, and largest particle heights, respectively. This nucleation box was located on the upper part of the nanoparticle, beneath the indent, and laterally symmetric to the indent. 


\subsubsection{Maximum number of nucleation attempts}

At each time step, the system repeatedly attempted to nucleate dislocations at randomly chosen sites and slip systems until the nucleation process was successful or if the number of attempts exceeded 5000. If the later criterion was met, the DDD simulation proceeded by one time step without nucleating a dislocation.

The DDD simulations were initialized with no dislocations in the nanoparticle, and the initial indenter depth was set to a starting value. Hence, we applied the stress field obtained from the FE simulation that was scaled by a time-dependent factor:

$\sigma_{\mathrm{app}}=\sigma_{\mathrm{FE}} \times f_{\mathrm{SF}} / \Delta \varepsilon_{0}$,

where $f_{\mathrm{SF}}$ is a stress factor and $\Delta \varepsilon_{0}$ is a reference strain. An initial value for the stress factor $f_{\mathrm{SF}_{0}}<1$ was chosen, so that the stress level remained below a threshold value for dislocation nucleation at the very beginning. Then, the scale factor was increased linearly with time, during which dislocation nucleation attempts were observed at each time step. The rate at which this factor increased was chosen to be small enough so that the first nucleation event occurred after about $10^{4}$ time steps.

Once a dislocation was nucleated, the stress levels were incremented in a manner that imposed constant plastic strain rate deformation on average. The plastic strain rate was calculated from the area swept by the dislocations over the whole simulated domain, and projected in the direction of the indentation. In all the simulations, the imposed strain rate was set to $\dot{\varepsilon}_{\text {imp }}=3 \times 10^{6} \mathrm{~s}^{-1}$. Consequently, the increase in the stress factor between two consecutive time steps was equal to

$\Delta f_{S F}=\left(\dot{\varepsilon}_{\mathrm{imp}}-\dot{\varepsilon}_{\mathrm{p}}\right) \Delta t$,

where $\dot{\varepsilon}_{\mathrm{p}}$ is the effective simulated plastic strain rate.

We note that the stress factor can increase or decrease after dislocation nucleation, depending on the plastic strain rate, in order to maintain the value imposed during the simulation. This algorithm results in an increase in the stress factor during the early stages of the plastic deformation. However, at the later stages, the increase in the stress factor decreases, and the stress factor may even become negative, according to Eq. (4), after an avalanche of dislocation nucleations.

\subsection{DDD simulation of a $116 \mathrm{~nm}$ high nanoparticle without cross-slip}

Nanoparticles of various sizes between $23 \mathrm{~nm}$ and $116 \mathrm{~nm}$ in height were indented. In the text that follows, we describe in detail the evolution of the dislocation structure in a $116 \mathrm{~nm}$ high nanoparticle. We first describe the results without incorporating the effect of dislocation cross-slip. Then, we examine the contribution of cross-slip to the results and the effect of size.

\subsubsection{Early stage nucleation}

The first nucleation occurs when the stress distribution is favorable for a particular slip system to nucleate a loop. In all simulations, the first dislocation nucleation occurs in the out-of-plane direction (Fig. 10a). After the first dislocation nucleation, another dislocation loop is rapidly nucleated in a different out-of-plane direction, as demonstrated in Fig. 10b. Both the loops expand by glide after the nucleation and interact while expanding, since their slip systems are non-
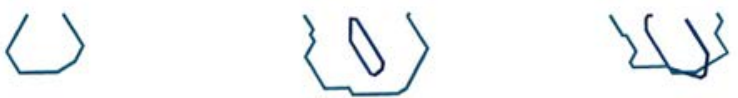

parallel. The nature of the interaction depends on the Burgers vectors of the nucleated loops. If the Burgers vectors are different, a junction will be formed. However, if the two dislocations have the same Burgers vector but opposite line senses, they participate in a collinear reaction, as has been reported based on the MD simulations. This collinear reaction between the two dislocations results in two v-shaped dislocations (Fig. 10c-d). These v-shaped dislocations are mobile and were found to be main carriers of plasticity in the DDD simulations.

\subsection{2. $V$-shaped dislocations and their interactions}

As the indentation proceeds, more dislocations are nucleated beneath the indent. Most of the dislocations are nucleated in the out-ofplane directions, and they mostly interact via collinear reactions, resulting in v-shaped dislocations. As a result, there is an increasing number of v-shaped dislocations forming in the three out-of-plane directions. The v-shaped dislocations are glissile, and their motion is restricted only by the point at the tip of the v-shaped configuration; the gliding occurs in the direction of the Burgers vector. As more of the vshaped dislocations are formed, they are increasingly pushed in the [1 011$]$, [ [ $\left.\begin{array}{lll}0 & 1\end{array}\right]$ or $\left[\begin{array}{lll}\overline{1} & 1 & 0\end{array}\right]$ directions towards the nanoparticle lateral surfaces and the bottom (see Supplementary Movie 3).

As a v-shaped dislocation glides away from the indent, both its ends travel on the upper surface. Upon reaching the edges of the nanoparticle, the end of the line transfers into the lateral facet. Owing to the strong line tension forces acting on the segment that is on the surface, the ends change their orientations and align themselves in a nearly perpendicular direction to the free surface. Consequently, the velocity of the ends on the surface is increased during the transition from the upper facet to the lateral ones.

While gliding away from the indent, some collinear reactions between the v-shaped dislocations can also occur. For example, a prismatic loop or a u-shaped dislocation (a dislocation made of 3 segments in three different glide planes, but with the same Burgers vectors) can be formed simply due to the collinear reactions between the dislocations nucleated in the out-of-plane directions. Several collinear reactions are described in Fig. 11 and in Supplementary Movie 4, where a prismatic loop is shown to "progress" in the out-of-plane direction through a series of collinear reactions involving consecutive dislocations in the out-of-plane direction. As a result, the prismatic loop still

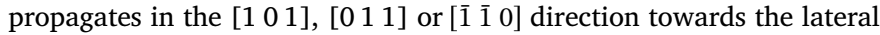
surfaces.

\subsubsection{Nucleation in the in-plane direction}

During the simulations, the nucleation of loops on planes parallel to the upper surface was also identified. These planes are expected to have an almost zero Schmid factor at the beginning of the simulations, hence, in almost all the simulations, dislocations in the in-plane direction were not observed at the beginning. However, the nucleation of out-of-plane dislocations stimulated the nucleation of loops parallel to the upper surface (see Supplementary Movie 3). Since the Schmid factor on these planes is low, the indenter stress alone was insufficient to expand the loops. However, their interaction with the other dislocations in the plastic zone allowed them to grow and, as the v-shaped dislocations glide laterally, these loops expand towards the lateral surfaces as well. Nevertheless, the contribution of the in-plane dislocation loops to the total plasticity was not found to be substantial. As we shall further (b) (c) (a)

to participate in a collinear reaction at their point of intersection. (d) After the interaction, two v-shaped dislocations are formed.
Fig. 10. Dislocation nucleation at the onset of plasticity in a $116 \mathrm{~nm}$ particle and an imposed plastic strain rate of $3 \times 10^{6} \mathrm{~s}^{-1}$. (a) An out-of-plane dislocation loop is nucleated beneath the indent that during growth touches the top free surface. (b) A second dislocation loop with the same Burgers vector is nucleated in another out-of-plane direction. (c) While expanding, both the dislocations are about

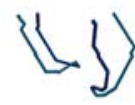

. 

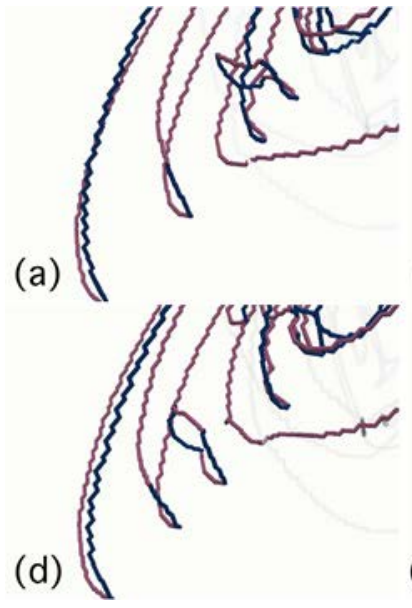

(b)

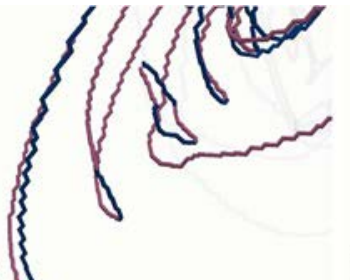

(e)

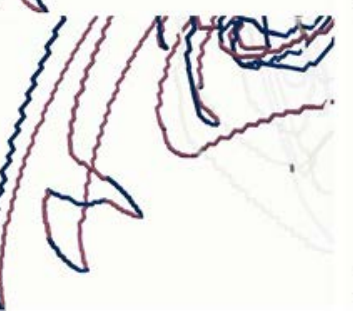

(c)

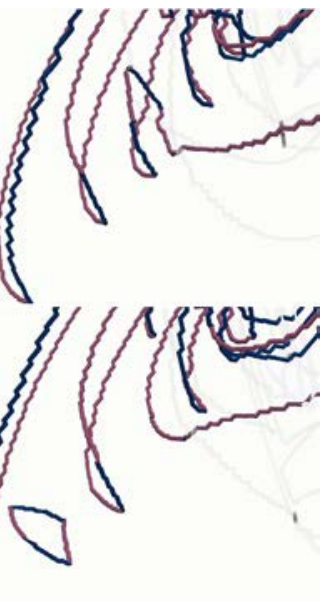

Fig. 11. Formation/annihilation of prismatic loops owing to multiple collinear reactions. (a)-(b) A prismatic loop participates in two collinear reactions with a v-shaped dislocation, and a new prismatic loop (second loop) is formed, leaving behind a v-shaped dislocation. (c)-(d) The newly formed prismatic loop participates in a collinear reaction with another dislocation, forming a new prismatic loop (third loop), leaving behind a dislocation. (e)-(f) The third prismatic loop participates in a collinear reaction with a u-shaped dislocation, which again results in a new prismatic loop further away from the indenter (see Supplementary Movie 4). reveal, this is not the case when cross-slip is introduced.

\subsection{Contribution of cross-slip}

In the simulations described above, cross-slip was omitted. However, cross-slip allows the screw segments to change slip planes and promotes additional interactions. Let us therefore repeat the DDD simulation of a $116 \mathrm{~nm}$ high nanoparticle with a cross-slip mechanism being active. Adding cross-slip does not change the dislocation mechanisms at the early stages of the deformation; the dislocations nucleated in the out-of-plane directions interact and form successive vshaped dislocations in the three out-of-plane directions. However, cross-slip of the screw dislocation segments converts the v-shaped dislocation into dislocation lines, which lie on more than two slip planes, e.g., u-shaped dislocations. These u-shaped dislocations glide away from the indent, similar to the v-shaped dislocations; in that sense, they contribute similarly to the deformation. However, this shape allows several additional interactions to take place.

For brevity, we will not detail here all the different possible interactions between the successive dislocations in the out-of-plane directions. We will just mention that it may convert v-shaped dislocations into u-shaped ones or even more complex configurations. However, one important contribution of cross-slip is to promote the formation of prismatic loops. Prismatic loops in the out-of-plane directions may be formed either via double cross-slip, as was reported previously [34], or by interactions between successive $\mathrm{v}$-shaped dislocations. The former mechanism is similar to the one observed in MD simulations of $\mathrm{Fe}$, where the cross-slip activation barrier is small [57], and the latter mechanism is demonstrated in Fig. 12. The upper parts of a v-shaped dislocation, which are of screw character, cross-slip and become a ushaped dislocation (Fig. 12a), which then annihilates with a segment of another u-shaped dislocation (Fig. 12b). As a result, a prismatic loop is pinched out and it glides in the out-of-plane direction, alongside the vshaped dislocations (Fig. 12c). We note that the motions of both the vshaped dislocations and the prismatic loops are restricted by points at the intersection of the slip planes, and they escape at the lateral surfaces

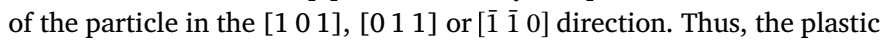
deformations expected with the motions of the v-shaped and u-shaped dislocations and the prismatic loops are similar.

Another contribution of cross-slip is to the in-plane dislocation loops. The screw parts along such loops can cross-slip into the out-ofplane directions and glide towards the upper surface to annihilate there. This process is demonstrated in Fig. 13. As a result, two half-prismatic loops (or a u-shaped dislocation) that glide in the in-plane directions are formed. The half-prismatic loops can also be formed by two collinear reactions between an in-plane loop and an open out-of-plane loop. As opposed to the nucleated in-plane loops, the half-prismatic loops easily glide laterally if there is no obstacle, which is expected to lead to surface deformation of the upper part of the nanoparticle.

To summarize the observations from the DDD simulation of a $116 \mathrm{~nm}$ high nanoparticle, dislocations were found to be nucleated on the four possible $\left\{\begin{array}{lll}1 & 1 & 1\end{array}\right\}$ planes. The dislocations in the out-of-plane directions rapidly formed $\mathrm{v}$-shaped and u-shaped dislocations and prismatic loops that glided along three slip directions towards the lower part of the nanoparticle. In addition, the dislocations that were nucleated parallel to the upper surface formed half-prismatic loops in the in-plane directions by collinear reactions or cross-slip. These half-prismatic loops glided laterally in the upper part of the nanoparticle. Some of the nucleated dislocations remained sessile beneath the indent, corresponding to the formation of dislocation junctions that helped in anchoring the plastic zone beneath the indent. Thus, the DDD simulation shows the interplay between the accumulation of dislocations inside the nanoparticle and their escape at the lateral surfaces. To understand the effect of size on the competition between the two mechanisms, we perform DDD simulations of nanoparticles of various sizes.

\subsection{Effect of nanoparticle size on plastic strain}

To examine the effect of size, three nanoparticles of different sizes (a)

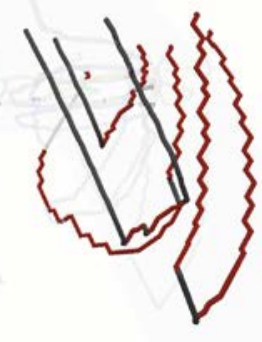

(b)

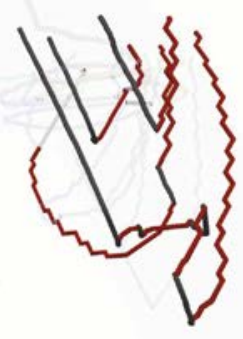

(c)
Fig. 12. Collinear reactions of two u-shaped dislocations. (a) One segment of one of two successive u-shaped dislocations glides towards the second one. (b) The first collinear reaction between two ushaped dislocations produces a v-shaped dislocation. (c) The second collinear reaction produces a prismatic loop; two successive collinear reactions of a ushaped dislocation may produce a prismatic loop. 
(a)

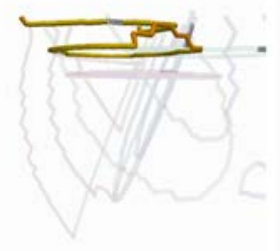

(b)

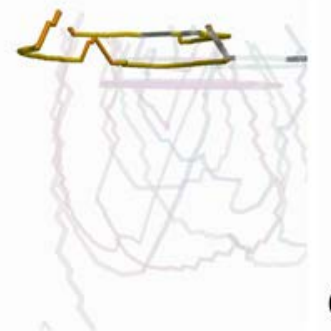

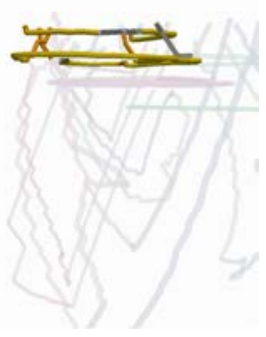

Fig. 13. Formation of half-prismatic loops. (a) A part of an in-plane dislocation loop cross-slips into an out-of-plane slip plane and glides towards the top surface. (b) The cross-slipped segment splits upon reaching the top surface, while another part of the loop starts cross-slipping. (c) The second cross-slipped segment splits at the top surface and, thus, creates two half-prismatic loops, among which one easily glides towards the lateral surfaces. were indented as part of DDD simulations: 23,69 and $116 \mathrm{~nm}$ in height. During the early stages of plasticity, a few out-of-plane dislocations were nucleated regardless of the nanoparticle size. This is an expected result, since the lateral dimensions or height does not substantially affect the nucleation conditions underneath the indent. Once the out-ofplane dislocations are nucleated, they expand and participate in collinear reactions to rapidly form $\mathrm{v}$-shaped dislocations. Independent of the nanoparticle size, these v-shaped configurations are highly mobile under the heterogeneous stress of the indenter. Owing to this higher mobility, they are strongly affected by the proximity of the lateral surfaces. Here, we note that the v-shaped dislocations need to cover fewer glide paths in smaller particles owing to the proximity of the lateral surfaces, compared with the larger ones. Consequently, as more $\mathrm{v}$-shaped dislocations are formed in the out-of-plane directions, those in the smallest particle escape easily, whereas, in the larger nanoparticles, they pile-up, as more v-shaped dislocations are formed beneath the indent. Moreover, in the smallest nanoparticle, a few nucleated halfloops can expand without reacting with another dislocation. When annihilating at the lateral surfaces, such dislocations convert into a straight line, gliding downwards in the out-of-plane direction and eventually depositing a dislocation at the impenetrable bottom of smaller particles (see Fig. 14a and Supplementary Movie 5).

In larger particles, nucleated dislocations interact to form v-shaped dislocations and prismatic loops before reaching the lateral surfaces (see Fig. 14b-c and Supplementary Movies 6 and 7 corresponding to 69 and $116 \mathrm{~nm}$ high nanoparticles, respectively). It is worth mentioning that it is almost impossible to form prismatic loops in the smaller particles owing to the scarcity of flight time and space. Since the dislocations in the larger particles are more prone to interact among themselves, cross-slip has a greater effect, compared with the smaller particles. After the first few out-of-plane dislocations are formed, inplane dislocations are nucleated, and they either form half-prismatic loops by cross-slip or expand in their original planes. While a pronounced effect of the lateral surfaces on the in-plane dislocations was not observed, their existence beneath the indent helps in forming a plastic zone. As can also be seen in Fig. 14, a certain portion of the dislocations form v-shaped dislocations or prismatic loops and glide away from the particle, whereas some bundle beneath the indent. The proportion of dislocations that escape is size-dependent.

Another indication of the effect of the lateral surfaces on starving the dislocations of the nanoparticle can be seen in Fig. 15. In this figure, the total dislocation length within the particle is plotted as a function of

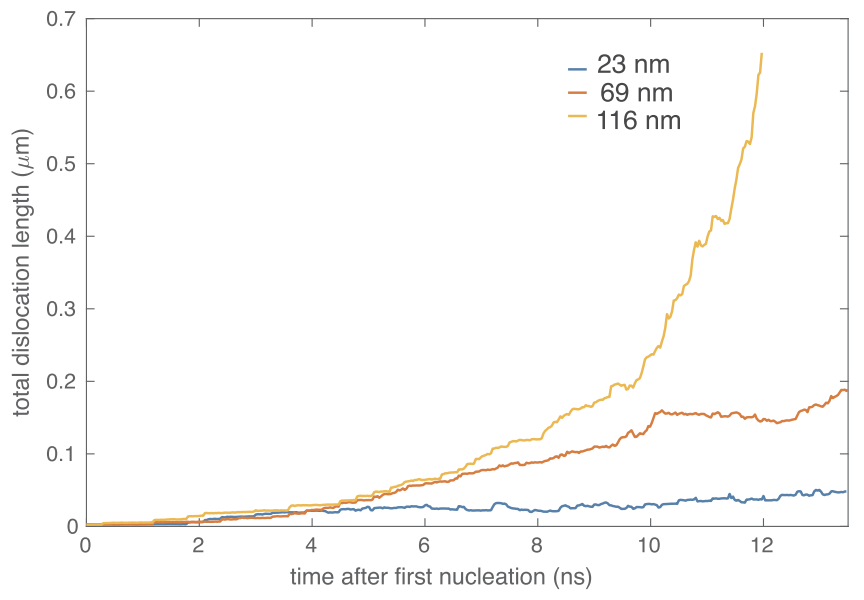

Fig. 15. Total dislocation lengths for different particle sizes as functions of time. All the simulations were performed at the constant imposed plastic strain rate of $3 \times 10^{6} \mathrm{~s}^{-1}$.

the time from the first nucleation event. Since all the simulations were performed at the same imposed plastic strain rate, the comparison also corresponds to the same indentation depth. One can see that at the early stages of the deformation, all the sizes yield similar increases in the dislocation length with time. The smallest particle deviates first from the curve when the first v-shaped dislocations formed escape from the nanoparticle (at about $4 \mathrm{~ns}$ after the first nucleation event). In what follows, the annihilation rate is large enough to lead to the lowest rate of increase in the total dislocation length. The intermediate-sized nanoparticle deviates from the largest particle at higher indentation depths, which corresponds to a time of approximately $7 \mathrm{~ns}$ after the first nucleation. At this time, we did not yet see dislocations escape from the nanoparticle, but the interaction of the mobile v-shaped dislocations allowed more collinear annihilation reactions to occur and a more rapid material transfer to the lateral surface. Finally, in the largest nanoparticles, the v-shaped dislocations in the plastic zone are kept localized near the indent. Consequently, the slopes of the curves shown in Fig. 15 are indicative of the increases in the dislocation lengths for a strain increment, i.e., they are indicative of strain hardening. Here, the sizedependent slope is mainly a result of the rate at which dislocations are depleted by annihilation at the free surfaces of nanoparticles of different sizes. The dislocation line storage rate calculated for the $116 \mathrm{~nm}$
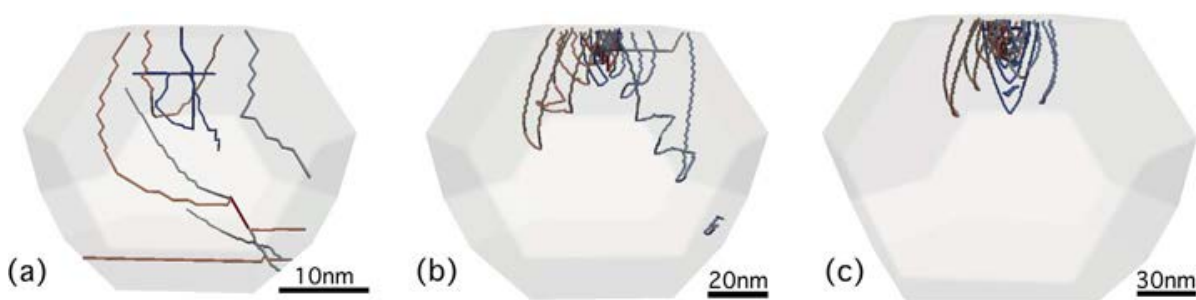

Fig. 14. Snapshots of the dislocation microstructures in 23 (a), 69 (b) and $116 \mathrm{~nm} \mathrm{(c)} \mathrm{high}$ nanoparticles at the same simulation time of $8.7 \mathrm{~ns}$ after the nucleation of the first dislocation. (a) Most of the dislocations in the $23 \mathrm{~nm}$ high particle escaped from the free surfaces. Some dislocations deposited at the bottom of the nanoparticle are reminisces of the nucleated dislocation loops that did not interact with the other dislocations, but rather interacted with the lateral surfaces. (b) In the $69 \mathrm{~nm}$

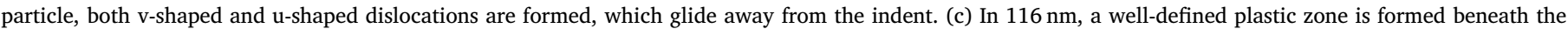
indent. The v-shaped or u-shaped dislocations participate in collinear reactions and form prismatic loops that glide towards the lateral surface. 
nanoparticle approaches the thin film solution, where dislocation depletion is controlled by collinear and cross-slip annihilations.

In summary, dislocations were found to nucleate beneath the indent and some escaped through the lateral surfaces. In the largest particle, a well-defined plastic zone was formed beneath the indent and a few dislocations escaped through the lateral surfaces, whereas, most of the dislocations escaped from the smallest particles and the plastic zone developed underneath the indent could not be well defined. In addition, some dislocations were deposited at the bottom of the smallest nanoparticle. Thus, different sizes of particles change the proximity of the lateral surfaces close to the plastic zone. For smaller particles, this proximity facilitates dislocations to escape from the specimen, and therefore, there is the possibility of interaction of dislocations with the lateral surfaces.

\section{Discussion and concluding remarks}

Both MD and DDD simulations demonstrate the role played by the lateral free surfaces in the formation of the plastic zone underneath the indent and the mechanisms by which dislocations are depleted from nanoparticles. There are, however, some differences in the mechanisms between the two simulation techniques. The MD simulations have shown that there are two dominant mechanisms, either in the out-ofplane direction or in the in-plane direction. The surface deformation observed at the lower part of the nanoparticle was a result of the motion of v-shaped dislocations and prismatic loops in the out-of-plane directions, whereas a pronounced deformation at the upper part was a result of severe dislocation multiplication following glissile reaction in the inplane direction. The formation of $\mathrm{v}$-shaped dislocations and prismatic loops was also observed in the DDD simulations, leading mostly to the depletion of the nucleated dislocations in the out-of-plane directions. However, the DDD simulation code employed here lacks the rules to model the multiplication processes that may be promoted by glissile junctions at the microscale [58]. In the DDD simulations, most of the inplane dislocation motion was a result of the nucleation of shear planar loops in the in-plane direction. Still, this feature emphasizes the main contribution of the glissile interactions noticed in the MD simulations and the possibility of deforming nanoparticles in the in-plane direction. Nonetheless, the fundamental results are the same, regardless of the mechanisms that allow dislocations to glide freely to free surfaces: if nucleated dislocations find a way, either via the formation of prismatic loops or through glissile interactions, to escape from the plastic zone, the percentage of dislocations depleted from the surface and not contributing to the formation of the plastic zone strongly depends on the size. As a result, we expect to see larger indentation forces for larger nanoparticles.

The simulations demonstrated the importance of collinear reactions on the evolution of the dislocation microstructure during nanoindentation. Collinear reactions led to the formation of v-shaped dislocations during the early stages of the indentation, and mainly allowed downward dislocation motion. This motion was even accelerated owing to the interactions among the piled-up v-shaped dislocations, which also allowed the formation of prismatic loops. The prismatic loops glided easily towards the lateral surfaces. In addition, cross-slip was also shown to be an important mechanism by which dislocations could escape from the plastic zone. Cross-slip allows the formation of prismatic loops in the out-of-plane directions and half-prismatic loops in the in-plane direction. It is interesting to note that the rate of formation of loops in the out-of-plane directions was found to depend on the indentation rate in the MD simulations, which leads us to believe that cross-slip of dislocation segments in a v-shaped structure is more pronounced at the indentation rate of $10 \mathrm{~m} / \mathrm{s}$ in the MD simulations.

In this work, we utilized a simple nucleation criterion. The use of such a criterion allows us to start with a pristine crystal, as opposed to other studies in which FR sources were distributed in the simulation cell. Such an approach also does not allow assuming a priori the nucleation sites and slip planes, as in the distribution of FR sources, and prevents interaction with artificially deposited sources. However, the nucleation of dislocations is a thermally activated process that involves the collective motion of a group of atoms, which cannot be easily captured to its full extent with the stress-dependent criterion used here (e.g., [59-62]). The exact details of the nucleation criterion may be important in the case of compression, for instance. When experimentally compressing nanoparticles, they yield a large strain burst at a stress that corresponds to the nucleation of a dislocation at the upper vertices. In this case, it is imperative that the exact conditions in which dislocations are nucleated are known to describe the strength of the nanoparticle under compression. In the case of nanoindentation, since nucleation is local underneath the indent and the main contribution to the plasticity is the interactions of dislocations amongst themselves and with the lateral surfaces, the quantitative details of the nucleation criterion are not crucial in our case. Comparison with the MD simulations indeed shows that nucleation in the DDD simulations occurred in the expected region beneath the indent, mostly in the out-of-plane directions, along with some nucleation in the in-plane direction. A more physically based nucleation criterion will allow in the future to quantify the onset-of-plasticity during nanoindentation in the DDD simulations, but we do not expect it to affect the main results of the present work.

In the DDD simulations performed as part of this work, the imposed elastic stress field was calculated independently of the DDD simulation boundary value problem. While this simplification allows us to obtain important information on the effect of size, it has several shortcomings. For instance, the image force solution used in the DDD simulations minimizes the forces of the dislocation segments that are touching the surface, but does not account for the forces on the dislocations approaching the free surface. This simplification on the image correction was assessed to introduce errors in the dislocation dynamics of the order of $10-20 \%$ [52]. However, our MD simulations show that when a $\mathrm{v}$-shaped dislocation expanded towards the free surface, it was only when its front reached the surface that the dislocation quickly annihilated there. Therefore, we believe that a more accurate consideration of the image forces will not yield a qualitatively different result and may only increase the size effect, since the dislocations in small particles will be more easily attracted to the lateral surfaces.

More profoundly, the indenter is not pushed into the nanoparticle, which deforms the 3D convex domain in the DDD simulation. Instead, an imposed effective plastic strain rate is defined based on the strain induced during dislocation motion. In this method, we allowed a gradual increase in the stress that permitted probing of the system in order to control the nucleation rate of dislocations as well as their glide and progress in the plastic zone. This simple means allows the control of stress in the DDD simulations through a unique FE calculation, which in turn allows us to compare between the different sizes for the same indentation depth. Nonetheless, while the use of a stress field from a FE calculation allowed the identification of important microstructural features, such as the dislocation nucleation beneath the indent and the effect of size on the dislocation structure, a more quantitative analysis is planned for the future by performing a fully coupled FE-DDD simulation with the Discrete-Continuous Model (DCM). This alternative modeling approach [63] will take into consideration additional properties such as the indentation rate and surface deformation during indentation, as well as accounting more accurately for the image forces. DCM simulations will also allow us to quantitatively examine the force-displacement curves obtained from the DDD simulations. 


\section{CRediT authorship contribution statement}

S. Roy: Methodology, Software, Validation, Formal analysis, Writing - original draft, Writing - review \& editing, Visualization. R. Gatti: Methodology, Software, Validation, Formal analysis, Writing review \& editing, Visualization, Supervision. B. Devincre: Conceptualization, Methodology, Software, Validation, Formal analysis, Resources, Writing - review \& editing, Visualization, Supervision. D. Mordehai: Conceptualization, Methodology, Software, Validation, Formal analysis, Resources, Writing - original draft, Writing - review \& editing, Visualization, Supervision, Funding acquisition.

\section{Acknowledgment}

This work was supported by Israel Science Foundation through Grants No. 1656/12 and 1521/16. S.R. and D.M. thank Dr. Avraham Dorogoy for his assistance with setting up the ABAQUS simulations.

Data availability

The raw/processed data required to reproduce these findings cannot be shared at this time owing to technical or time limitations.

\section{References}

[1] W.D. Nix, J.R. Greer, G. Feng, E.T. Lilleodden, Deformation at the nanometer and micrometer length scales: effects of strain gradients and dislocation starvation, Thin Solid Films 515 (6) (2007) 3152-3157.

[2] T. Zhu, J. Li, Ultra-strength materials, Prog. Mater Sci. 55 (7) (2010) 710-757.

[3] M.D. Uchic, D.M. Dimiduk, J.N. Florando, W.D. Nix, Sample dimensions influence strength and crystal plasticity, Science 305 (5686) (2004) 986-989.

[4] M. Ramos, L. Ortiz-Jordan, A. Hurtado-Macias, S. Flores, J. Elizalde-Galindo, C. Rocha, B. Torres, M. Zarei-Chaleshtori, R.R. Chianelli, Hardness and elastic modulus on six-fold symmetry gold nanoparticles, Materials 6 (1) (2013) 198-205.

[5] A. Gouldstone, N. Chollacoop, M. Dao, J. Li, A.M. Minor, Y.-L. Shen, Indentation across size scales and disciplines: Recent developments in experimentation and modeling, Acta Mater. 55 (12) (2007) 4015-4039.

[6] Y.M. Soifer, A. Verdyan, M. Kazakevich, E. Rabkin, Edge effect during nanoindentation of thin copper films, Mater. Lett. 59 (11) (2005) 1434-1438.

[7] D.K. Ward, D. Farkas, J. Lian, W.A. Curtin, J. Wang, K.-S. Kim, Y. Qi, Engineering size-scaling of plastic deformation in nanoscale asperities, Proc. Nat. Acad. Sci. 106 (24) (2009) 9580-9585.

[8] D. Ge, A.M. Minor, E.A. Stach, J.W. Morris Jr., Size effects in the nanoindentation of silicon at ambient temperature, Phil. Mag. 86 (25-26) (2006) 4069-4080.

[9] Y. Choi, K.J. Van Vliet, J. Li, S. Suresh, Size effects on the onset of plastic deformation during nanoindentation of thin films and patterned lines, J. Appl. Phys. 94 (9) (2003) 6050-6058.

[10] M.A. Zeeshan, D. Esqué-de Los Ojos, P. Castro-Hartmann, M. Guerrero, J. Nogués, S. Suriñach, M.D. Baró, B.J. Nelson, S. Pané, E. Pellicer, et al., Electrochemically synthesized amorphous and crystalline nanowires: dissimilar nanomechanical behavior in comparison with homologous flat films, Nanoscale 8 (3) (2016) 1344-1351.

[11] J. Li, A.H.W. Ngan, P. Gumbsch, Atomistic modeling of mechanical behavior, Acta Mater. 51 (19) (2003) 5711-5742.

[12] J.R. Greer, W.D. Nix, Nanoscale gold pillars strengthened through dislocation starvation, Phys. Rev. B 73 (24) (2006) 245410.

[13] S.H. Oh, M. Legros, D. Kiener, G. Dehm, In situ observation of dislocation nucleation and escape in a submicrometre aluminium single crystal, Nature Mater. 8 (2) (2009) 95-100.

[14] M.F. Horstemeyer, M.I. Baskes, S.J. Plimpton, Length scale and time scale effects on the plastic flow of fcc metals, Acta Mater. 49 (20) (2001) 4363-4374.

[15] V. Dupont, F. Sansoz, Molecular dynamics study of crystal plasticity during nanoindentation in ni nanowires, J. Mater. Res. 24 (3) (2009) 948-956, https://doi. org/10.1557/jmr.2009.0103.

[16] E.L. Wood, T. Avant, G.S. Kim, S.K. Lee, Z. Burchman, J.M. Hughes, F. Sansoz, Size effects in bimetallic nickel-gold nanowires: Insight from atomic force microscopy nanoindentation, Acta Mater. 66 (2014) 32-43.

[17] D. Chrobak, N. Tymiak, A. Beaber, O. Ugurlu, W.W. Gerberich, R. Nowak, Deconfinement leads to changes in the nanoscale plasticity of silicon, Nature Nanotechnol. 6 (8) (2011) 480-484.

[18] D. Mordehai, M. Kazakevich, D.J. Srolovitz, E. Rabkin, Nanoindentation size effect in single-crystal nanoparticles and thin films: a comparative experimental and simulation study, Acta Mater. 59 (6) (2011) 2309-2321.
[19] J.-I. Zhang, S. Zaefferer, D. Raabe, A study on the geometry of dislocation patterns in the surrounding of nanoindents in a TWIP steel using electron channeling contrast imaging and discrete dislocation dynamics simulations, Mater. Sci. Eng. A 636 (2015) 231-242.

[20] P.C. Wo, A.H.W. Ngan, Y.L. Chiu, Tem measurement of nanoindentation plastic zones in ni3al, Scripta Mater. 55 (6) (2006) 557-560.

[21] E. Van der Giessen, A. Needleman, Discrete dislocation plasticity: a simple planar model, Modell. Simul. Mater. Sci. Eng. 3 (5) (1995) 689.

[22] H.G.M. Kreuzer, R. Pippan, Discrete dislocation simulation of nanoindentation: the effect of moving conditions and indenter shape, Mater. Sci. Eng.: A 387 (2004) 254-256.

[23] J. Segurado, J. Llorca, An analysis of the size effect on void growth in single crystals using discrete dislocation dynamics, Acta Mater. 57 (5) (2009) 1427-1436.

[24] G.R. Canova, Y. Bréchet, L.B. Kubin, B. Devincre, V. Pontikis, M. Condat, 3d simulation of dislocation motion on a lattice: application to the yield surface of single crystals, Solid State Phenomena, vol. 35, Trans Tech Publ, 1993, pp. 101-106.

[25] K. Yasuda, K. Shinohara, M. Yamada, M. Kutsuwada, C. Kinoshita, A new method for evaluating stress-strain properties of metals using ultra-microhardness technique, J. Nucl. Mater. 187 (2) (1992) 109-116.

[26] L.E. Shilkrot, R.E. Miller, W.A. Curtin, Multiscale plasticity modeling: coupled atomistics and discrete dislocation mechanics, J. Mech. Phys. Solids 52 (4) (2004) 755-787.

[27] M.C. Fivel, C.F. Robertson, G.R. Canova, L. Boulanger, Three-dimensional modeling of indent-induced plastic zone at a mesoscale, Acta Mater. 46 (17) (1998) 6183-6194.

[28] Z. Wang, N. Ghoniem, S. Swaminarayan, R. LeSar, A parallel algorithm for 3d dislocation dynamics, J. Comput. Phys. 219 (2) (2006) 608-621.

[29] J.A. El-Awady, H. Fan, A.M. Hussein, Advances in discrete dislocation dynamics modeling of size-affected plasticity, Multiscale Materials Modeling for Nanomechanics, Springer, 2016, pp. 337-371.

[30] H.-J. Chang, H.H. Han, M.C. Fivel, Multiscale Modelling of Nanoindentation, Key Eng. Mater. 345-346 (2007) 925-930.

[31] H.-J. Chang, M. Fivel, D. Rodney, M. Verdier, Multiscale modelling of indentation in fcc metals: from atomic to continuum, C.R. Phys. 11 (3-4) (2010) 285-292.

[32] M.C. Fivel, C.F. Robertson, G.R. Canova, L. Boulanger, Three-dimensional modeling of indent-induced plastic zone at a mesoscale, Acta Mater. 46 (17) (1998) 6183-6194.

[33] G. Po, M.S. Mohamed, T. Crosby, C. Erel, A. El-Azab, N. Ghoniem, Recent progress in discrete dislocation dynamics and its applications to micro plasticity, JOM: J. Miner., Metals, Mater. Soc. 66 (2014) 2108-2120.

[34] J. Gagel, D. Weygand, P. Gumbsch, Formation of extended prismatic dislocation structures under indentation, Acta Mater. 111 (2016) 399-406.

[35] L.B. Munday, J.C. Crone, J. Knap, The role of free surfaces on the formation of prismatic dislocation loops, Scripta Mater. 103 (2015) 65-68.

[36] L.B. Munday, J.C. Crone, J. Knap, Prismatic and helical dislocation loop generation from defects, Acta Mater. 103 (2016) 217-228.

[37] F. Liu, Z.-L. Liu, P. Lin, Z. Zhuang, Numerical investigations of helical dislocations based on coupled glide-climb model, Int. J. Plast. 92 (2017) 2-18.

[38] S. Plimpton, Fast parallel algorithms for short-range molecular dynamics, J. Comput. Phys. 117 (1) (1995) 1-19.

[39] G. Wulff, Zur frage der geschwindigkeit des wachstums und der auflosung der kristall achen, Z. Kristallogr 34 (1901) 449-530.

[40] G. Grochola, S.P. Russo, I.K. Snook, On fitting a gold embedded atom method potential using the force matching method, J. Chem. Phys. 123 (20) (2005) 204719

[41] A. Stukowski, K. Albe, Extracting dislocations and non-dislocation crystal defects from atomistic simulation data, Modell. Simul. Mater. Sci. Eng. 18 (2010).

[42] Y. Feruz, D. Mordehai, Towards a universal size-dependent strength of face-centered cubic nanoparticles, Acta Mater. 103 (2016) 433-441.

[43] H.Y. Liang, C.H. Woo, H. Huang, A.H.W. Ngan, T. Yu, Dislocation nucleation in the initial stage during nanoindentation, Philos. Mag. 83 (31-34) (2003) 3609-3622.

[44] S. Shao, H.M. Zbib, I.N. Mastorakos, D.F. Bahr, Deformation mechanisms, size effects, and strain hardening in nanoscale metallic multilayers under nanoindentation, J. Appl. Phys. 112 (4) (2012) 044307.

[45] J. Li, K.J. Van Vliet, T. Zhu, S. Yip, S. Suresh, Atomistic mechanisms governing elastic limit and incipient plasticity in crystals, Nature 418 (6895) (2002) 307-310.

[46] K.J. Van Vliet, J. Li, T. Zhu, S. Yip, S. Suresh, Quantifying the early stages of plasticity through nanoscale experiments and simulations, Phys. Rev. B 67 (10) (2003) 104105

[47] B. Devincre, R. Madec, G. Monnet, S. Queyreau, R. Gatti, and L. Kubin. Modeling crystal plasticity with dislocation dynamics simulations: The 'micromegas' code. Mechanics of Nano-objects, pages 81-100, 2011.

[48] B. Devincre, V. Pontikis, Y. Brechet, G. Canova, M. Condat, L. Kubin, Three-dimensional simulations of plastic flow in crystals, Microscopic simulations of complex hydrodynamic phenomena, Springer, 1992, pp. 413-423.

[49] X.H. Liu, K.W. Schwarz, Modelling of dislocations intersecting a free surface, Modelling Simul. Mater. Sci. Eng. 13 (2005) 1233-1247.

[50] H. Tang, K.W. Schwarz, H.D. Espinosa, Dislocation escape-related size effects in single-crystal micropillars under uniaxial compression, Acta Mater. 55 (5) (2007) 1607-1616.

[51] M. Grydlik, F. Boioli, H. Groiss, R. Gatti, M. Brehm, F. Montalenti, B. Devincre, F. Schäffler, L. Miglio, Misfit dislocation gettering by substrate pit-patterning in sige films on si (0 0 1), Appl. Phys. Lett. 101 (1) (2012) 013119

[52] X.H. Liu, F.M. Ross, K.W. Schwarz, Dislocated epitaxial islands, Phys. Rev. Lett. 85 (19) (2000) 4088.

[53] D. Hull, J.D. Bacon, Introduction to Dislocations, Butterworth-Heinemann, 2001.

[54] D. Mordehai, S.-W. Lee, B. Backes, D.J. Srolovitz, W.D. Nix, E. Rabkin, Size effect in 
compression of single-crystal gold microparticles, Acta Mater. 59 (13) (2011) 5202-5215.

[55] Z.-J. Wang, Q.-J. Li, Y. Li, L.-C. Huang, L. Lu, M. Dao, J. Li, E. Ma, S. Suresh, Z.W. Shan, Sliding of coherent twin boundaries, Nature Commun. 8 (1) (2017) 1108.

[56] N.M. Ghoniem, E.P. Busso, N. Kioussis, H. Huang, Multiscale modelling of nanomechanics and micromechanics: an overview, Philos. Mag. 83 (31-34) (2003) 3475-3528.

[57] R. Kositski, D. Mordehai, Depinning-controlled plastic deformation during nanoindentation of BCC iron thin films and nanoparticles, Acta Mater. 90 (2015) 370-379.

[58] M. Stricker, D. Weygand, Dislocation multiplication mechanisms -glissile junctions and their role on the plastic deformation at the microscale, Acta Mater. 99 (2015) $130-139$.
[59] T. Zhu, J. Li, K.J. Van Vliet, S. Ogata, S. Yip, S. Suresh, Predictive modeling of nanoindentation-induced homogeneous dislocation nucleation in copper, J. Mech. Phys. Solids 52 (3) (2004) 691-724.

[60] R.E. Miller, A. Acharya, A stress-gradient based criterion for dislocation nucleation in crystals, J. Mech. Phys. Solids 52 (7) (2004) 1507-1525.

[61] R.E. Miller, D. Rodney, On the nonlocal nature of dislocation nucleation during nanoindentation, J. Mech. Phys. Solids 56 (4) (2008) 1203-1223.

[62] A. Garg, A. Acharya, C.E. Maloney, A study of conditions for dislocation nucleation in coarser-than-atomistic scale models, J. Mech. Phys. Solids 75 (2015) 76-92.

[63] O. Jamond, R. Gatti, A. Roos, B. Devincre, Consistent formulation for the discretecontinuous model: Improving complex dislocation dynamics simulations, Int. J. Plast. 80 (2016) 19-37. 